\title{
Álgebra Temprana como herramienta de análisis y comprensión de problemas aritméticos en primaria
}

\section{Early Algebra as a tool for analysis and understanding of arithmetic problems in primary}

DOI: http://dx.doi.org/10.17981/cultedusoc.9.1.2018.01

Recibido: Noviembre 5 de 2017 / Aceptado: Febrero 22 de 2018

Ana Medrano y Rosa del Carmen Flores-Macías

Universidad Nacional Autónoma de México (México) lrcfm@unam.mx

Para citar este artículo

Medrano, A. y Flores-Macías, R. (2018). Álgebra Temprana como herramienta de análisis y comprensión de problemas aritméticos en primaria. Cultura. Educación y Sociedad 9 (1), 9-26. DOI: http://dx.doi.org/10.17981/cultedusoc.9.1.2018.01

\section{Resumen}

En el presente estudió, se evaluó el efecto de una secuencia instruccional, basada en Instrucción de Ampliación de Esquemas (Fuchs, Fuchs, Prentice, Hamlett, Finelli \& Courey, 2004) sobre el desempeño en la solución de problemas que admiten tanto una solución algebraica como aritmética y problemas algebraicos. Se planteó un diseño pre test - post test con grupo control. Participaron 27 estudiantes de tercer grado que conformaron el grupo experimental (GE) y 21 estudiantes de cuarto grado que conformaron el grupo control (GC). Los resultados sugieren un efecto diferencial positivo en los estudiantes del GE. Se discute sobre las bondades de implementar este tipo de secuencias instruccionales en la educación básica.

Palabras Clave: Solución de problemas, transferencia, algebra temprana, representación.

\begin{abstract}
The purpose of this article was to evaluate the effect of an instructional sequence based on Instruction of Extension of Schemes over the performance in the solution and representation of arithmetic problems of Total (Fuchs, Fuchs, Prentice, Hamlett, Finelli \& Courey, 2004) and algebraic (Schliemann, Carraher, Brizuela and Jones, 2011). A pretest - posttest design with control group was proposed. 27 third grade students formed the experimental group (EG) and 21 fourth grade students formed the control group (CG). The results suggest a positive differential effect on the group that was exposed to the instructional sequence (EG) on the solution and algebraic representation of total arithmetic problems, as well as a positive differential effect in solving algebraic problems. We argument about of implementing this type of instructional sequences in elementary school are discussed.
\end{abstract}

Keywords: Word-problem solution, transfer, early algebra, representation. 


\section{Introducción}

Desde hace un par de décadas ha ganado relevancia la búsqueda de alternativas educativas que beneficien a todos los estudiantes de educación básica; la preocupación principal es que la educación promueva una mejor calidad de vida de los educandos y de la sociedad en su conjunto. En este sentido, se han orientados los esfuerzos de la investigación educativa en la búsqueda de alternativas educativas que fortalezcan el aprendizaje.

En particular, una preocupación ha sido que el aprendizaje de las matemáticas sea la base para continuar con el aprendizaje de otros conocimientos y sea una herramienta de pensamiento para resolver diferentes situaciones cotidianas que vive el alumnado preparándolos para que comprendan y apliquen conocimientos y habilidades en diferentes situaciones tanto dentro como fuera del aula.

Sin embargo, en los estudiantes mexicanos, se ha observado un pobre desempeño. En particular, se documentan dificultades para plantear, formular, interpretar y resolver problemas matemáticos vinculados a situaciones cotidianas. El Instituto Nacional para la Evaluación de la Educación (INEE) (2013) informa que en una escala de 0 a 5 , el $56 \%$ de los estudiantes se encuentran entre el nivel 0 y 1 . Los datos presentados en la prueba EXCALE-10 (INEE, 2013) muestran que los alumnos mexicanos de segundo y tercer grado de primaria presentan un déficit en la solución de problemas aritméticos. Señalan que 7 de cada 10 estudiantes son incapaces de encontrar una solución correcta a problemas aritméticos que requieren conocimientos elementales. Por su parte el Programa para la Evaluación Internacional de los Estudiantes (PISA, por sus siglas en inglés) (2009) reporta que sólo el 5\% de los estudiantes mexicanos muestran un razonamiento matemático avanzado en el dominio de las operaciones formales y simbólicas. Finalmente, el Tercer Estudio Regional Comparativo y Explicativo (TERCE) (2013) reporta que sólo el $20 \%$ de los estudiantes mexicanos se ubica en el nivel más alto (IV). Los resultados anteriores evidencian una falta de comprensión de las matemáticas y altos índices de reprobación que se acentúan cuando se introduce el álgebra.

La solución de problemas (aritméticos para la primaria, y algebraicos para la secundaria), constituye uno de los ejes principales del programa de matemáticas en México (Secretaria de Educación Pública, 2011); considerando los resultados de las diferentes evaluaciones antes citadas, se puede afirmar que los alumnos no aprenden a solucionar problemas mediante razonamientos centrados en el análisis de las relaciones y conceptuaciones matemáticas y a partir de la elaboración de sus propias representaciones; más bien, han aprendido a solucionarlos aplicando un algoritmo a partir de la comprensión superficial de los problemas y de la memorización de ciertas rutinas.

La consecuencia de estas carencias en el aprendizaje es que los alumnos no reconocen las relaciones matemáticas implicadas en el problema, no pueden generar estrategias eficientes para seguir un razonamiento y tienen 
dificultades para dar un significado apropiado a los algoritmos considerando el contexto del problema (Flores, 2005), lo que limita sus posibilidades para adaptarse y actuar en diferentes situaciones de su vida diaria que demandan una comprensión de conceptos y procedimientos matemáticos así como el desarrollo de sus capacidad de reflexión lógica necesaria para crear modelos que les lleven a explicar, predecir y modificar su realidad (Alsina, 2010). En resumen, sus experiencias de aprendizaje son limitadas y sus conocimientos deficientes.

Ante este panorama, se puede sostener que la enseñanza de la matemática dentro de las aulas mexicanas se ha alejado del pensamiento crítico y analítico, así como de los ámbitos de la vida real y del establecimiento de relaciones con otras áreas de conocimiento. La alternativa que se propone es enseñar las matemáticas en contextos en los que el estudiante comprenda y aplique conceptos y principios matemáticos al solucionar problemas, empleando un pensamiento estratégico durante el proceso de solución, para finalmente, transferir los conocimientos a situaciones novedosas y de la vida diaria. En este contexto es que se plantea como alternativa la enseñanza temprana del álgebra.

\section{El Álgebra Temprana como vía alterna en el aprendizaje de la solución de problemas}

Una opción educativa que pretende generar una enseñanza profunda e integradora de la matemática, y que podría coadyuvar a superar la situación descrita líneas atrás, es el Álge- bra Temprana (AT) (EA por sus siglas en inglés). Este movimiento educativo se inicia en la Unión Soviética con Davydov (1995) con su teoría de la actividad de aprendizaje y el desarrollo del aprendizaje. Él señala que los estudiantes necesitan asimilar el conocimiento teórico mientras que conocen el proceso por el cual se origina, lo que fortalece que resuelvan problemas mientras se centran en el principio general de su construcción. Dichos planteamientos son retomados y ampliados por James Kaput (2008) en Estados Unidos.

Davydov (1995) propuso que la construcción del pensamiento algebraico debe iniciar en los estudiantes de grados elementales, fomentando con ello la generalidad en el pensamiento. Sostiene que si se ofrece a los estudiantes herramientas culturales apropiadas (i.e. diagramas y anotaciones), los estudiantes podrán hacer más de lo que tradicionalmente se ha supuesto, es decir, se puede potenciar que atiendan a la estructura y relaciones implicadas en cada situación matemática. Desde este punto de vista, no se percibe una distinción entre la aritmética y el álgebra, ver las matemáticas desde ese enfoque favorece el desarrollo conceptual de situaciones matemáticamente cada vez más complejas desde edades muy tempranas (Blanton y Kaput, 2005; 2011). En Estados Unidos, esta propuesta educativa surge como alternativa al pobre desempeño que de los alumnos en pruebas internacionales (i.e. PISA), así como respuesta a los señalamientos de Consejo Nacional de Maestros de Matemáticas (2000; 2015) (NCTM por sus siglas en inglés). 
En línea con lo anterior, en años recientes surgió la propuesta educativa Instrucción de Ampliación de Esquemas (en inglés Schema-Broadening Instruction $S B I$ ) que tiene como propósito enseñar a analizar y resolver tres diferentes tipos de problemas aritméticos (combinación, de transformación, entre otros) introduciendo ecuaciones algebraicas como herramienta de análisis y representación de la estructura del problema.

Los principales representantes de la propuesta de Instrucción de Ampliación de Esquemas (IBE) son Fuchs, Fuchs, Prentice, Hamlett, Finelli y Courey (2004) y Fuchs, Zumeta, Finelli, Powell, Seethaler, Hamlett y Fuchs (2010). Ellos consideran una categorización de los problemas de acuerdo a su estructura subyacente, definida por las relaciones implicadas (total, diferencia y cambio). La identificación de la estructura del problema orienta al alumno para reconocer si pertenece a una categoría ya conocida y por tanto generaliza el proceso de solución o si se trata de una estructura nueva e inicia la búsqueda de una solución adecuada.

Para promover la comprensión y generalización de la estructura subyacente a cada tipo de problema matemático, Fuchs et al. (2010) presentan al alumno una representación genérica (i.e. $\mathrm{P} 1+\mathrm{P} 2=\mathrm{T}$ ), cuyos elementos son remplazados con la información proporcionada en la narración del problema e incorporan la literal " $x$ " para indicar el lugar de la información faltante, conformando con ello una ecuación algebraica (i.e. $8+\mathrm{x}=15$ ). Lo anterior facilita el reconocimiento de las características de cada tipo de problema, así como, de la categoría a la que pertenece un problema.

En el primer estudio que realizaron Fuchs, Seethaler, Powell, Fuchs, Hamlett y Fletcher (2008) participaron treinta y cinco estudiantes de tercer grado de primaria, los cuales fueron seleccionados en función de su desempeño en una prueba de lectura y otra de matemáticas. Los estudiantes que obtuvieron bajo desempeño en ambas pruebas fueron seleccionados y asignados de manera aleatoria al grupo experimental o a al grupo control. Ambos grupos contaron con enseñanza general en solución de problemas guiada por el maestro, en la cual se enseñó un conjunto de reglas en solución de problemas.

Al grupo experimental se le enseñó mediante tutorías individuales la Instrucción de Ampliación de Esquemas. Las sesiones se llevaron a cabo tres veces por semana con una duración de veinte a treinta minutos durante doce semanas. El objetivo de las tutorías fue que los estudiantes aprendieran a resolver los problemas que se describen a continuación.

- En los problemas de Total se combinan dos cantidades para formar una más grande. Este tipo de problemas refleja una relación de magnitud, siendo su representación $\mathrm{P} 1+\mathrm{P} 2=\mathrm{T}$ (parte uno más parte dos igual al total). Un ejemplo de este tipo de problema es: hay 51 niños y 47 niñas en tercer grado de primaria, ¿cuántos alumnos de tercer grado hay en total? Ecuación algebraica: $51+$ $47=\mathrm{x}$ 
- En los problemas de Diferencia se comparan dos cantidades considerando su magnitud. Su representación es $\mathrm{CM}-\mathrm{CP}=\mathrm{D}$ (cantidad mayor menos cantidad menor igual a la diferencia). Un ejemplo es, durante el día de campo, los niños comieron 54 pastelitos y 32 hamburguesas. ¿Cuántos pastelitos más que hamburguesas comieron los niños? Representación algebraica: $54-32=\mathrm{x}$

- Los problemas de Cambio (incremento y decremento) se caracterizan por el aumento o disminución de una cantidad, en estos problemas se reflejan transformaciones temporales. Su representación es: $\mathrm{SI}+/-\mathrm{C}=\mathrm{Sf}$ (situación inicial más/menos cambio igual a situación final). Un ejemplo de este tipo de problema es: algunas aves estaban en el comedero. Entonces vinieron 2 más. Ahora hay 5. ¿Cuántas aves estaban en el comedero al principio? Representación algebraica: $\mathrm{x}+2=3$

En estos tres tipos de problema, la incógnita puede ocupar cualquier elemento de la ecuación. (i.e. $51+\mathrm{x}=73$; $\mathrm{x}+25=73 ; 67+34=\mathrm{x}$ ).

Primero, los estudiantes aprendieron a identificar y representar con una ecuación problemas en una versión sencilla, posteriormente se enfrentaron a problemas novedosos que variaban con base en tres criterios diferentes: a) con información irrelevante; b) con información complementaria (i.e. tablas, figuras); c) con cantidades mayores. Posteriormente, se enseñó a resolver las ecuaciones conformadas con la información de cada problema.
La enseñanza se realizó durante tres sesiones semanales de treinta minutos a lo largo de doce semanas. Los resultados indicaron que el grupo experimental mostró un desempeño significativamente mejor en la solución de problemas en comparación con el grupo control.

Fuchs et al. (2010) realizaron un segundo estudio, emplearon la misma estrategia instruccional con el mismo tipo de problemas pero, a diferencia de la investigación previa en la que se impartió tutoría individual, en ésta, la enseñanza fue grupal. Participaron doscientos setenta estudiantes de segundo grado de primaria, asignados a uno de los dos grupos: experimental y control. Ambos recibieron enseñanza en habilidades básicas y estrategias generales para solucionar problemas, la cual pone énfasis en el acercamiento meta-cognitivo guiado por preguntas que ayudan en la comprensión para la solución de problemas narrativos. El grupo experimental además participó en la Instrucción de Ampliación de Esquemas. Los autores evaluaron y compararon el desempeño de los estudiantes ante dos diferentes pruebas. Antes de la Instrucción de Ampliación de Esquemas se evaluó la habilidad del cálculo y la comprensión y solución de problemas narrativos con un solo dígito, después de la Instrucción de Ampliación de Esquemas, se evaluó la comprensión y solución de problemas con información faltante en alguna de las tres diferentes partes de la ecuación y las habilidades básicas. Los resultados indicaron que el grupo experimental tuvo un mejor desempeño en la solución de proble- 
mas, el 58\% representó el problema mediante una ecuación algebraica. De ese 58\%, el $95.3 \%$ representó la ecuación de manera correcta. Los autores concluyen que la Instrucción de Ampliación de Esquemas favoreció el desempeño al solucionar problemas, además de que un poco más de la mitad empleó ecuaciones algebraicas para solucionarlos.

Un aspecto relevante que se señalan los autores es que la instrucción favoreció la solución de problemas cuando la incógnita se localizó en el primer elemento del primer miembro de la ecuación $(\mathrm{x}+6=15)$ y en el segundo miembro de la ecuación $(9+6=\mathrm{x})$; sin embargo, para los estudiantes fue complicado resolver las ecuaciones cuando la incógnita se encontró en el segundo elemento del primer miembro de la ecuación $(9+x=15)$. Los autores consideran que estos datos se contraponen a los reportados por otras investigaciones, donde la mayor dificultad se encuentra en la primera posición de la ecuación y proponen dar un mayor énfasis a la enseñanza en la solución de ecuaciones, así como, al desarrollo de estrategias de enseñanza y aprendizaje mediante las cuales los estudiantes puedan comprender y abstraer la estructura subyacente de diferentes tipos de problemas.

Las investigaciones de Fuchs y cols. (2008; 2010) sugieren que todos los estudiantes pueden beneficiarse de la Instrucción de Ampliación de Esquemas puesto que la ecuación les apoya para identificar la información importante del problema, así como, la relación implicada, y para representar la estructura que define cada tipo de problema. En este sentido, la ecua- ción funciona como un andamio que los estudiantes utilizan para ubicar la información relevante del problema y llegar a la solución. Los autores resaltan que emplear ecuaciones algebraicas para representar la estructura subyacente de diferentes tipos de problemas contribuye a dar coherencia y profundidad a las matemáticas, facilitando con ello que el estudiante establezca un puente entre la aritmética y el álgebra desde edades tempranas.

No obstante, los resultados antes comentados y del esfuerzo importante que se realiza desde esta propuesta para atender los señalamientos de organismos como el NCTM, la propuesta de Instrucción de Ampliación de Esquemas enfrenta en su versión actual, algunas limitantes. Primera, falta de referentes - criterios - claros para diferenciar la complejidad de cada problema. Si bien los autores señalan que incorporan información complementaria y/o relevante como tablas y gráficos, agregan información irrelevante en la narración del problema y ubicar la incógnita en un lugar diferente al conjunto total complejizan el problema, no indican en qué medida se complejiza o cuál de todos esos cambios representa una mayor demanda cognitiva para el niño; tampoco señalan si al interior de cada una de las variantes se encuentra un gradiente de complejidad, en resumen, no se analiza cuál es la implicación cognitiva de estas modificaciones que se realizan en el problema.

Segundo, los estudiantes que participaron en las diferentes investigaciones, presentan, en algunos casos, diferencias relacionadas con el aprovechamiento y rendimiento académico, 
aspecto que lleva a los autores a mencionar y clasificar a los participantes en dos grupos, con dificultades y sin dificultades de aprendizaje. A pesar de esta distinción, los resultados presentados en las diferentes investigaciones no se reportan manteniendo dicha distinción. Tampoco se realizan comparaciones entre el aprovechamiento de la Instrucción de Ampliación de Esquemas atendiendo a tales características.

Considerando que Fuchs y cols. emplean ecuaciones tipo en la propuesta didáctica para solucionar problemas aritméticos, que una ecuación algebraica representa la estructura subyacente y las relaciones de un tipo de problema y que ya se ha evaluado la transferencia de la ecuación como estructura subyacente a problemas aritméticos más complejos, es plausible suponer que la Instrucción de Ampliación de Esquemas puede promover la comprensión, representación y solución de problemas diferentes a los aritméticos, tales como los relacionales y algebraicos.

Para explorar empíricamente lo anterior y considerando las dificultades reportadas con la representación algebraica y la comprensión de la incógnita en el segundo elemento del primer miembro de la ecuación, en la presente investigación se analizó el efecto de una secuencia instruccional basada en Instrucción de Ampliación de Esquemas sobre el desempeño en la solución y representación de problemas aritméticos de total (Fuchs, Fuchs, Prentice, Hamlett, Finelli \& Courey, 2004) y algebraicos (Schliemann, Carraher, Brizuela y Jones, 2011).

\section{Método}

\section{Participantes}

Cuarenta y ocho estudiantes de una escuela primaria pública del estado de México. Veintisiete inscritos al tercer grado de primaria con edad promedio de 8 años y veintiún estudiantes inscritos en cuarto grado con edad promedio de 9 años de edad. Los estudiantes de tercer grado conformaron el grupo experimental (GE), mientras que los estudiantes de cuarto grado formaron parte del grupo control (GC). Los participantes fueron seleccionados de manera intencional no probabilística.

\section{Materiales}

Lápices, gomas, sacapuntas, plumones de diferente color, pintarrón y pizarrón.

\section{Aparatos e instrumentos}

- Computadora personal con sistema operativo Microsoft Windows 8.

- Video proyector marca HP.

- Cartas descriptivas para llevar a cabo la instrucción Basada en Esquemas, elaboradas ex profeso para la investigación.

- Dos evaluaciones en solución de problemas, equivalentes en el contenido y complejidad (ejemplos de los problemas algebraicos y de Total empleado se encuentran en el apéndice 1 y anexo 1). Ambas se validaron mediante "jueceo de expertos". Las evaluaciones estuvieron conformadas por quince diferentes problemas. De estos, doce fueron del tipo total y tres algebraicos. 
Los primeros variaron en complejidad considerando el lugar que ocupó la incógnita (se presentó en cualquiera de los elementos que conforman los dos miembros de la ecuación i.e. $\mathrm{x}+9=15 ; 6+\mathrm{x}=15$; $6+9=x)$, las cantidades empleadas (decenas o centenas) y el contexto de los números (conteo, uso de los números y medición). En todos los problemas los datos numéricos se refirieron a enteros (ver anexo 2 , ejemplos de problemas)

- Cuadernillo de trabajo impreso con los problemas de la secuencia instruccional, uno por alumno del GE.

\section{Escenario}

Las sesiones se llevaron a cabo en un salón de clases amueblado con bancas dobles para el total de los estudiantes. Ventilación natural e iluminación natural y artificial.

\section{Diseño}

El diseño empleado fue pre test-post test con grupo control.

\section{Variables}

Independiente: exposición a la secuencia instruccional.

Dependiente: Tipo de problemas presentados en las evaluaciones, a saber, problemas de total y algebraicos. Para evaluar se consideró el número de aciertos y el tipo de simbolización empleada (aritmética o algebraica).

$\mathrm{Al}$ igual que la literatura realizada por Fuchs y colaboradores, se con- sidera una simbolización algebraica cuando se incorpora la literal " $x$ " para denotar una cantidad incógnita en algún lugar de la representación del problema.

\section{Procedimiento}

El procedimiento constó de tres condiciones: a) Pre test, b) exposición a la secuencia instruccional basada en la Instrucción de Ampliación de Esquemas y c) Post test.

Tanto el pre-test como el post-test fueron idénticos para los dos grupos. A continuación, se describen las tres condiciones del estudio:

Pre test. Esta evaluación se realizó en la primera sesión y fue igual para los dos grupos. La evaluación consistió en leer y solucionar quince diferentes problemas. De estos, doce fueron del tipo total y tres algebraicos. El orden de los problemas fue aleatorizado.

Instrucción de Ampliación de Esquemas. Las sesiones se llevaron los días lunes, miércoles y viernes durante una hora diaria, hasta cubrir un total de treinta horas. Se siguieron los lineamientos expuestos por los autores de la propuesta. La primera sesión se destinó a que los alumnos dieran un significado a la noción de incógnita y lo asociaran a la literal " $\mathrm{x}$ ". Las siguientes sesiones se trabajaron con problemas de Total con la incógnita en el Conjunto Total (CT), es decir, del lado derecho del signo de igualdad $(9+6=\mathrm{x})$; se enseñó que los problemas se pueden representar mediante una ecuación que sintetiza y organiza la información del problema y que la parte del problema desconocida se representaría con una "x" en la ecuación matemática $(12+$ 
$13=\mathrm{x}$ ); luego, se trabajó con problemas de Total, presentando la incógnita en el segundo elemento del primer miembro de la ecuación $(9+x=15)$. Una vez generada la ecuación que relaciona los elementos importantes implicados en este tipo de problemas, se pidió a los estudiantes que encontrarán el valor de la incógnita. Después de las sesiones dedicadas a explorar y analizar los diferentes procedimientos para localizar el valor de la incógnita, las sesiones se dedicaron a modelar la solución algebraica formal de la ecuación generada, empleando la concatenación de términos y el despeje de la incógnita. En las siguientes sesiones, se presentaron problemas de Total, ubicando la incógnita en cualquier miembro de la ecuación algebraica $(9+6=\mathrm{x} ; \mathrm{x}+6=15 ; 9$ $+\mathrm{x}=15$ ). Todas las soluciones $\mathrm{y}$ abordajes se comentaron de manera grupal. En las últimas sesiones, se pidió a los estudiantes inventar y solucionar problemas de Total que debían de expresar las relaciones implicadas en la ecuación que se les proporcionaba.

Post test. Se aplicó al día siguiente de terminar con la implementación de la secuencia instruccional. Esta evaluación fue igual para los dos grupos y consistió en leer y solucionar quince diferentes problemas, doce del tipo total y tres algebraicos. Los problemas que conformaron el post-test difirieron de los del pre-test únicamente en la narración de la situación problema.

\section{Resultados}

Los resultados y los análisis comparativos se presentan en función de los aciertos obtenidos ante cada tipo de problema, así mismo, se discuten las diferencias en el tipo de simbolización empleada en cada problema (aritmética y algebraica). El análisis se realiza tanto al interior de cada grupo como comparando el desempeño de los dos grupos.

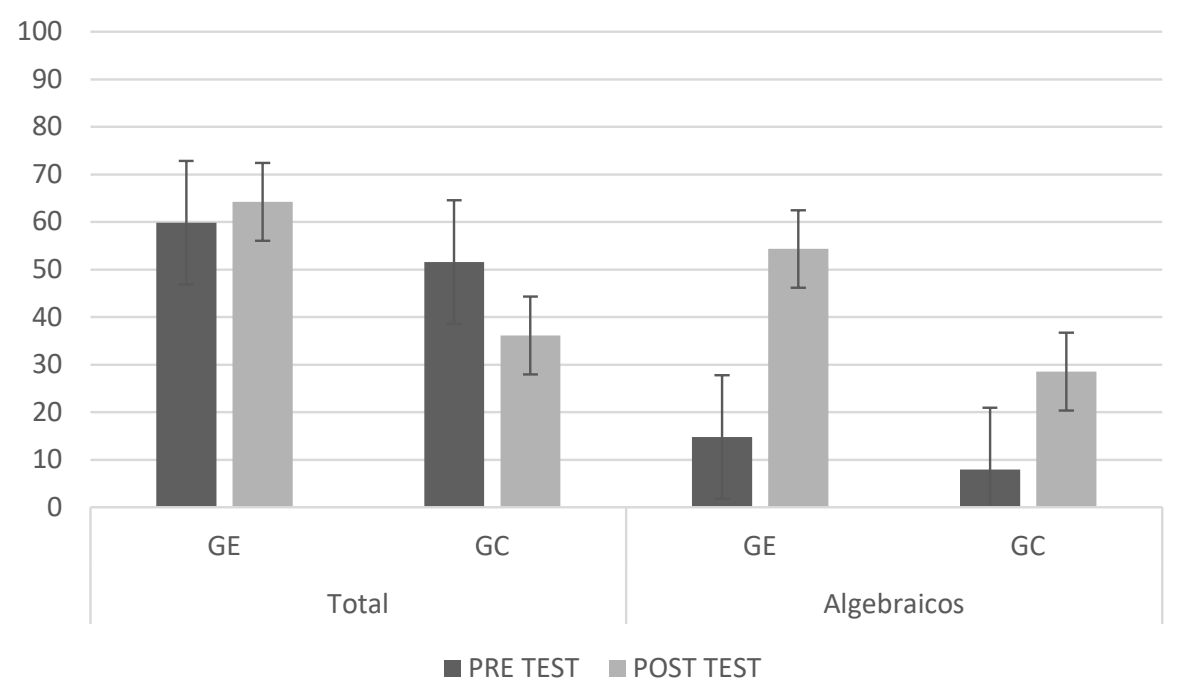

Fig. 1. Porcentaje de respuestas correctas obtenido por los dos grupos ante los dos tipos de problemas presentados en las dos diferentes evaluaciones. Fuente: Elaboración propia. 
La figura 1 muestra el porcentaje de respuestas correctas obtenido por los dos grupos ante los dos tipos de problemas que conformaron las dos evaluaciones. Se observa que el Grupo Experimental $(G E)$ incrementó el porcentaje de respuestas correctas en el post test en ambos tipos de problemas (Total y algebraicos). El incremento ante los problemas de Total fue de 4.3 puntos porcentuales, pasando de $59.9 \%$ a $64.2 \%$, mientras que para los problemas algebraicos el incremento fue de 39.5 puntos porcentuales, pasando de $14.8 \%$ a 54.3\%. Para identificar si las diferencias observadas entre las dos evaluaciones son significativas, se realizó una prueba $T$ de student para muestras relacionadas. Se encontró que sí hay diferencias estadísticamente significativas ante los problemas de total $\mathrm{t}(\mathrm{gl}=26)=-3.731 ; \mathrm{p}=.00) \mathrm{y}$ ante los problemas algebraicos $\mathrm{t}(\mathrm{gl}=26)$ $=-7.832 ; \mathrm{p}=.00$

Dado que las evaluaciones estuvieron conformadas por dos diferentes ti- pos de problemas y dado que al interior de los problemas de Total, los problemas presentaron la incógnita en diferentes partes de la ecuación, a saber, en el conjunto total $(12+13=\mathrm{x})$, en la parte $1(\mathrm{x}$ $+13=25)$ o en la parte $2(12+\mathrm{x}=25)$, incluyendo, problemas con tres partes 0 sumandos (i.e. $13+x+8=33$ ), se analizó el desempeño de los estudiantes ante cada problema presentado.

La figura 2 muestra que la mayor ganancia obtenida en el desempeño del GE se observó en los problemas algebraicos, incrementando 39.5 puntos porcentuales del pre-test al post-test, pasando de $14.8 \%$ a $54.3 \%$. La segunda mayor ganancia observada se presentó ante los problemas de Total con la incógnita en la parte 1 (i.e. $x+13=25$ ), el porcentaje obtenido en el pre-test incrementó 29.6 puntos porcentuales, pasando de $39.5 \%$ a $69.1 \%$. En lo que refiere al desempeño mostrado por el GC, éste, con excepción de los problemas algebraicos, disminuye su porcentaje de respuestas correctas del

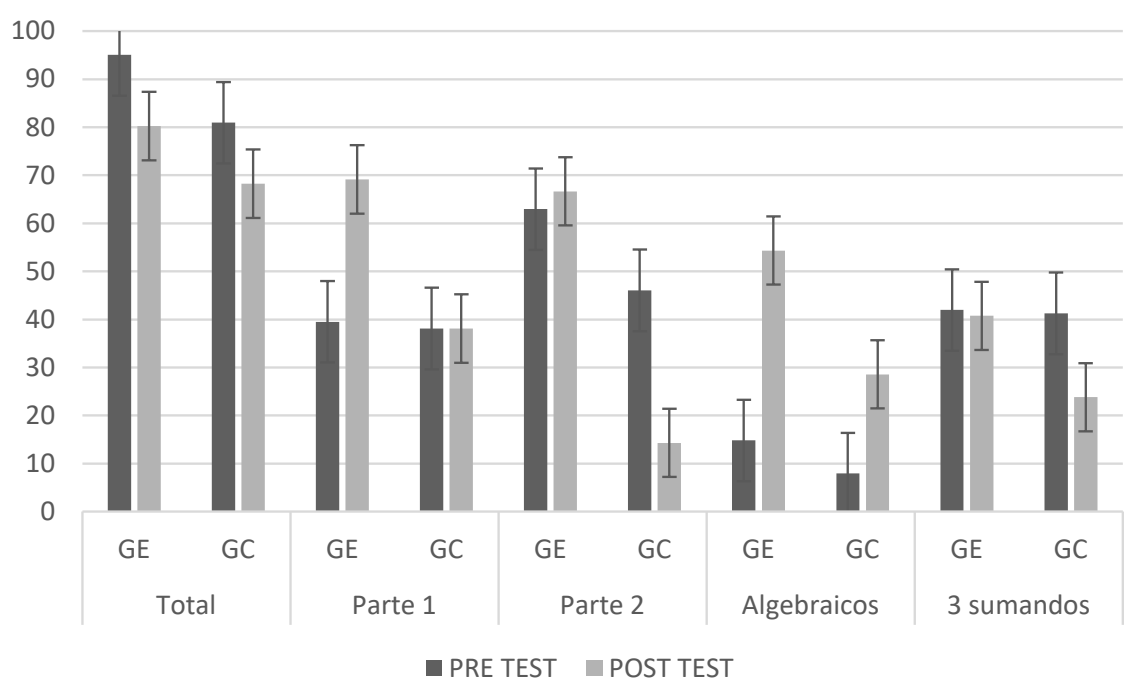

Fig. 2. Porcentaje de respuestas correctas obtenido por los grupos ante los diferentes problemas presentados en las dos evaluaciones.

Fuente: Elaboración propia. 
pre-test al post-test. Con la finalidad de detectar si las diferencias observadas los diferentes problemas (diferenciados por el lugar de la incógnita) son estadísticamente significativas, se realizó la prueba estadística t de student para muestras relacionadas encontrando que las diferencias son estadísticamente significativas para los problemas que presentan la incógnita en la parte $1 \mathrm{t}$ $(\mathrm{gl}=26)=-3.693 ; \mathrm{p}=.00)$ y para los problemas algebraicos $\mathrm{t}(\mathrm{gl}=26)=-7.832$; $\mathrm{p}=.00$

Un segundo propósito, de la investigación, fue identificar el tipo de representaciones que emplearon los estudiantes. La figura 3 muestra que, en el pre-test tanto para los problemas de Total como para los algebraicos, los estudiantes de ambos grupos realizaron únicamente representaciones aritméticas, cabe señalar que no en todos los casos fueron correctas. En lo que respecta a la post-evaluación, los estudiantes del GE diversificaron sus representacio- nes, incorporando lo aprendido durante la secuencia instruccional. Para los problemas de Total las simbolizaciones aritméticas obtuvieron un $44.8 \%$, mientras que las algebraicas obtuvieron un $55.2 \%$.

Es pertinente señalar que al igual que Fuchs, et al. (2004; 2008; 2010), en esta investigación se considera una simbolización algebraica cuando se identifican las siguientes tres condiciones: a) se reconoce que hay una incógnita; b) se señaliza la incógnita con la literal x y c) se reconoce la estructura del tipo de problema donde se ubica la incógnita. En la post evaluación para los problemas algebraicos, las simbolizaciones aritméticas obtuvieron un $77.8 \%$ mientras que las simbolizaciones algebraicas un $22.2 \%$. El grupo control únicamente empleó representaciones aritméticas. Cabe mencionar que todas las representaciones aritméticas se caracterizaron por el uso y relación entre cantidades específicas implicadas en el problema.

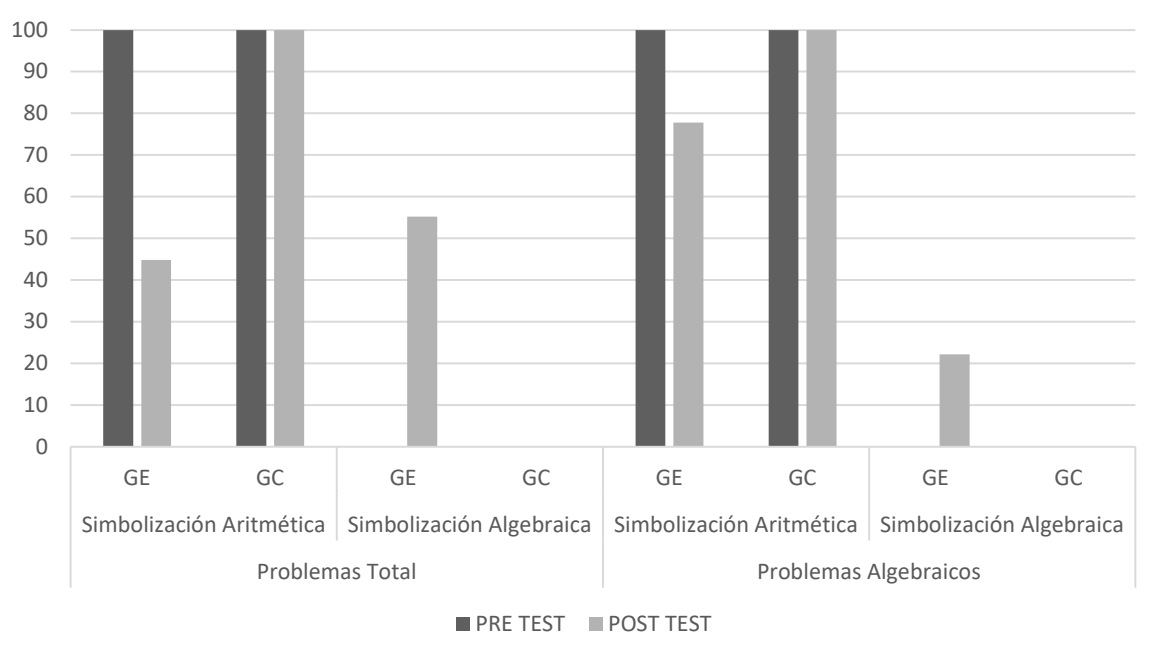

Fig. 3. Porcentaje de simbolizaciones realizadas por los dos grupos, ante cada tipo de problema presentado en las evaluaciones.

Fuente: Elaboración propia. 


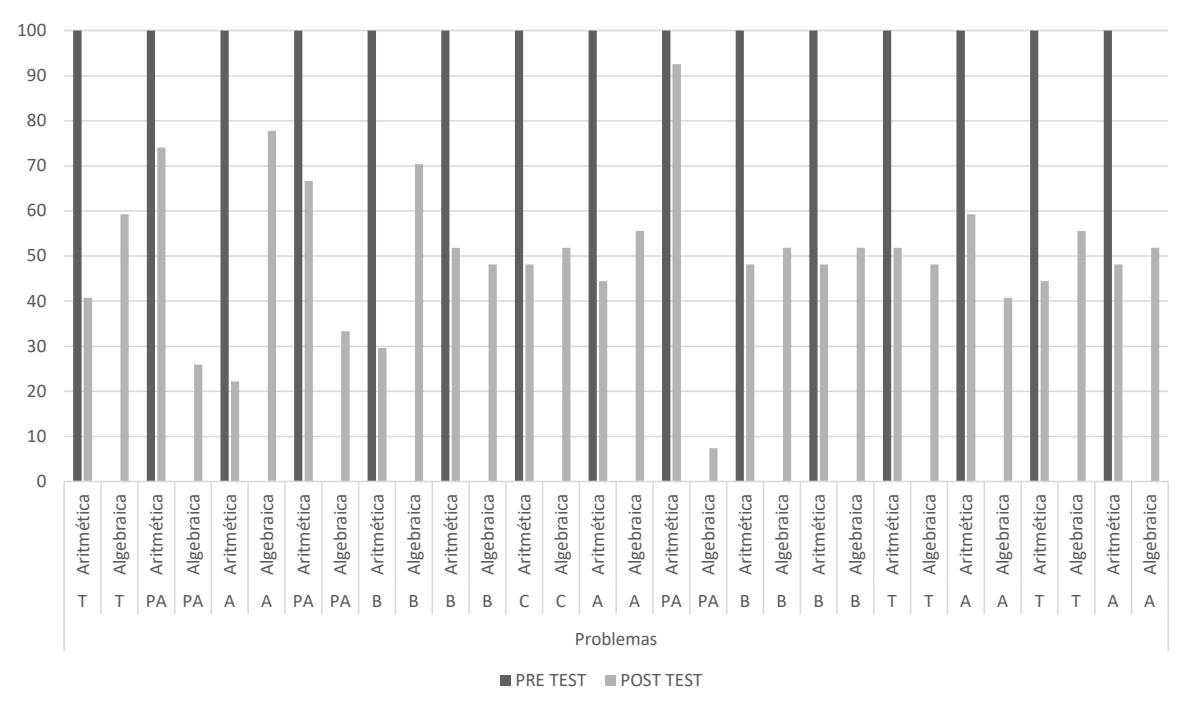

Fig. 4. Porcentaje de simbolización realizada por el GE ante cada tipo de problema. Fuente: Elaboración propia.

Finalmente, la figura 4 muestra las simbolizaciones realizadas por el GE ante los quince problemas que conformaron las dos evaluaciones. De manera general se observa que, en la post-evaluación, ante los problemas algebraicos el mayor porcentaje de simbolización fue aritmético, mientras que para los problemas de Total el mayor porcentaje de simbolización fue algebraica.

Con base en los resultados antes presentados, se puede resumir que el 81.5\% de los estudiantes del Grupo Experimental reconocieron una incógnita dentro de las relaciones implicadas en los problemas de Total y la representaron con una ecuación empleando la literal " $\mathrm{x}$ " acorde con la estructura del tipo de problema. Referente a los problemas de tipo Algebraico, el 55.5\% de los estudiantes emplearon una ecuación y reconocieron una o más incógnitas dentro de las relaciones implicadas en los problemas y la o las representaron con la literal " $x$ ".

\section{Discusión}

El propósito de la investigación fue evaluar el efecto de una secuencia instruccional basada en Instrucción de Ampliación de Esquemas sobre el desempeño en la solución y representación de problemas aritméticos de total (Fuchs, Fuchs, Prentice, Hamlett, Finelli \& Courey, 2004) y algebraicos (Schliemann, Carraher, Brizuela y Jones, 2011).

Los datos presentados aportan evidencia sobre la asimilación y significación del conocimiento promovido mediante la Instrucción de Ampliación de Esquemas, así como de la transferencia de conocimientos a problemas matemáticos con características completamente diferentes (problemas algebraicos) a los problemas de Total, aspecto que no se había evaluado previamente.

El análisis de resultados permite sostener que la exposición a la Instrucción de Ampliación de Esquemas, en concordancia con la literatura del área, favoreció 
la comprensión y solución de problemas de Total, pero también la transferencia de conocimientos para comprender y solucionar problemas algebraicos haciendo uso de literales (en nuestro caso de la $x$ ) para simbolizar la incógnita. Estos datos permiten afirmar que es plausible y útil en la primaria incorporar elementos del álgebra, que tradicionalmente se han enseñado hasta la secundaria; se mostró que facilitan el análisis y comprensión de las relaciones implicadas en los diferentes problemas, contribuyendo a una solución efectiva.

La solución de problemas de Total y algebraicos basada en Instrucción de Ampliación de Esquemas puede contribuir a la significación y familiarización de conceptos y simbolizaciones propias del álgebra (i.e. uso de literales, significación de la literal como incógnita, etc.). Sin embargo, la presente propuesta educativa no facilitó el tránsito y significación a la noción de literal como variable y como función, ya que los problemas planteados, trabajados durante la secuencia instruccional se enfocan en encontrar un valor particular y específico. Este aspecto impide que los estudiantes exploren y, por ende, signifiquen los conceptos algebraicos, como la variable, incorporados en los problemas algebraicos lo que pudo llevar a los alumnos del GE a simbolizar y manipular este tipo de problemas de forma aritmética.

Una alternativa que permitiría subsanar dicha limitante, sería incorporar situaciones problema que permitan al estudiante explorar y significar diferentes conceptos algebraicos, como por ejemplo problemas que involucren un pensamiento relacional o funcional, prescindiendo de valores particulares (i.e. Bernardo va a tener su fiesta de cumpleaños y quiere asegurarse de que todos sus amigos se pueden sentar. Él tiene mesas cuadradas. En una mesa cuadrada se pueden sentar 4 personas. ¿En 3 mesas cuadradas cuántas personas se pueden sentar?, En 10 mesas ¿cuántas personas se pueden sentar?, en cualquier número de mesas, ¿cuántas personas se pueden sentar?). Lo anterior contribuiría a que el estudiante se aleje de situaciones que para su solución sólo requiere razonar con valores específicos, transitando al pensamiento relacional y funcional.

Por otra parte la secuencia instruccional no facilitó el despeje de la literal para obtener una solución algebraica. Si bien los alumnos emplearon una ecuación para sintetizar y relacionar las diferentes relaciones implicadas en los dos diferentes tipos de problemas presentados en las evaluaciones, al resolver el problema, los niños obtuvieron el valor de la incógnita mediante una operación aritmética.

En lo que refiere a la simbolización, se puede decir que la ecuación es una herramienta que permite organizar la información del problema, así como el proceso de solución. La representación es un elemento esencial en la comprensión (Vygotsky, 2009) ya que funge como una herramienta de mediación entre el problema y la acción del estudiante, A partir de la representación es que se comprende el problema y las relaciones implicadas, para posteriormente, construir una solución (Schliemann, Carraher y Brizuela, 2011).

Los estudiantes aprendieron que la representación se modifica conforme las características de los problemas cambian. Este punto hace plausible suponer que los estudiantes significaron ciertas nociones durante la secuencia instruccional, haciendo uso de ellas en situaciones novedosas y cambiantes, evidencia de esto es que las diferentes simbolizacio- 
nes que realizaron los alumnos del GE se ajustan a las relaciones que establece cada tipo de problema, ubicando la incógnita en un lugar distinto del total, para los problemas de Total e incorporando más de una literal para los problemas algebraicos.

En lo que refiere al análisis de las simbolizaciones en el GE, se puede comentar que las aritméticas incluyen dentro de sus elementos las relaciones implicadas en el problema, tal cual fueron enunciadas en la narración. Este tipo de simbolización explicita de manera directa y clara la operación aritmética que se debe realizar para obtener el resultado correcto a la interrogante plateada en el problema (i.e. $6+9=15$ ). Por el contrario, las representaciones algebraicas, características de la conformación de una ecuación al detectar una incógnita y señalarla con la literal $x$, no explicitan de forma directa y clara la operación que se debe realizar para obtener el valor de la incógnita (i.e. $23+\mathrm{x}=54$ ). En este caso, el alumno debe inferir, a partir de su conocimiento sobre conceptos matemáticos la relación recíproca - inversa - a la operación estipulada en la ecuación, en este caso la relación recíproca entre la suma y la resta. Una vez que se comprende esto, se puede realizar la operación y obtener el valor de la incógnita que hace verdadera la relación enunciada en la ecuación.

En ambas formas de simbolización se plasman las relaciones implicadas en el problema, pero la simbolización algebraica implica una comprensión distinta de las relaciones, ya que demanda el razonamiento y comprensión de conceptos y elementos no evidentes en la simbolización aritmética (i.e. relación recíproca). Por ejemplo, en la ecuación $23+\mathrm{x}=54$, el alumno aprende que no se trata de sumar la primera y segunda parte, sino que se trata de establecer una relación inversa recíproca entre los elementos conocidos para llegar al valor de x (Flores, 2006). Este hecho, que a primera vista puede resultar simple, estructura de forma cualitativamente distinta la comprensión de las relaciones implicadas en el problema y, por ende, del razonamiento que se pone en juego para llegar a la solución y al valor dela incógnita. Las simbolizaciones de problemas mediante una ecuación son un salto cualitativo importante en el aprendizaje de los estudiantes, ya que el análisis que realizan permite la incorporación de otros elementos y conceptos matemáticos, así como la comprensión de las relaciones de manera general.

La simbolización de los alumnos, para los diferentes problemas, hace evidente que ellos recurren a las herramientas con las que cuentan para analizar y sintetizar las relaciones implicadas. La manipulación que se dé a las diferentes simbolizaciones está en función de la historia interactiva que se tenga con ciertas prácticas y conocimientos. Aspecto que hace de suma importancia promover diferentes historias de simbolización y manipulación matemática en la comprensión y solución de diferentes tipos de problemas.

En relación con lo anterior, los resultados expuestos sugieren que, aunque los estudiantes trabajaron con las mismas situaciones problema y se expusieron a la misma secuencia instruccional, cada estudiante se apropió de forma distinta de los conceptos enseñados y matematizó de forma diferente la situación problema presentada, lo que en última instancia posibilitó los diferentes tipos de solución, representación y simbolización.

La obtención de un porcentaje menor en las representaciones algebraicas en comparación con las aritméticas, en lo que a problemas Algebraicos refiere, 
puede deberse principalmente a dos razones. La primera, es que las relaciones implicadas en los problemas algebraicos fueron completamente nuevas para los estudiantes, ya que la secuencia instruccional basada en la Instrucción de Ampliación de Esquemas únicamente trabajó con problemas de Total. La segunda razón refiere a las características de las relaciones implicadas en los problemas y de la respuesta misma que soluciona el problema algebraico. Es decir, los problemas algebraicos no demandan que los estudiantes obtengan una única y particular respuesta, de hecho, la respuesta que se pide, en algunos casos no es numérica.

Los problemas algebraicos enuncian y establecen relaciones entre los elementos del problema, por ejemplo, en el siguiente problema algebraico Bárbara y Juana tuvieron su fiesta de cumpleaños el mismo día. Bárbara recibió 7 regalos de sus amigos, y Juana también recibió 7 regalos de sus amigos. Cuando cada fiesta habia terminado, las niñas pasaron un tiempo con sus respectivas familias y recibieron aún más regalos. Bárbara recibió 6 regalos más de su familia y Juana recibió 3 regalos más de su familia. Al final del día, ipiensas que Juana recibió la misma cantidad de regalos que Bárbara o piensas que una recibió más regalos que la otra?, la pregunta que se plantea no se responde únicamente con una cantidad o valor específico. Si el niño atiende los valores de los regalos que recibieron Bárbara y Juana, podrá decir que Bárbara tiene más porque en su casa recibió un mayor número de regalos, a diferencia de Juana. Siendo esta una respuesta correcta a la problemática planteada. Este dato es entendible dado que los alumnos del GE hasta antes de la realización del estudio no contaban con ningún conoci- miento sobre el análisis y solución de problemas con estas características.

En resumen, la secuencia instruccional llevó al GE a emplear conocimientos y herramientas diferentes a los algoritmos. La propuesta del estudio es una extrapolación de la planteada por Fuchs et al. (2004; 2008; 2010), ya que además de representar las relaciones implicadas en el problema mediante una ecuación y presentar la incógnita en diferentes lugares de la ecuación, también evalúa la comprensión y representación de dos problemas diferentes. Los primeros incluyen tres sumandos $(12+\mathrm{x}+21=55)$, mientras que en los segundos predomina la comprensión de literales y la relación de diferentes relaciones enunciadas en el problema, dejando de lado la manipulación de los números, estos se denominaron algebraicos.

Esta propuesta junto con la hecha por Fuchs y colaboradores, son alternativas que pueden servir de guía para favorecer la comprensión y solución de diferentes problemas matemáticos, así como para relacionar el álgebra con la aritmética desde los primeros años de educación básica.

Un punto que queda pendiente y que se retomará en investigaciones futuras es el relacionado con la manipulación tanto formal como natural que los estudiantes hacen de la ecuación que elaboran para simbolizar las diferentes relaciones implicadas en un determinado problema. Esto es importante porque a pesar de que la secuencia instruccional basada en Instrucción de Ampliación de Esquemas brindó enseñanza explícita en el manejo formal de las ecuaciones, es decir, en el despeje de la incógnita, los participantes decidieron manipularlas y obtener el valor de la incógnita mediante el uso de la aritmética. 


\section{Agradecimientos}

La primera autora agradece al Consejo Nacional de Ciencia y Tecnología (CONACyT) de México el apoyo otorgado para la presente investigación mediante la beca nacional otorgada al becario con el número de registro 478960.

\section{Referencias}

Alsina, A. (2012). Hacia un enfoque globalizado de la educación matemática en las primeras edades. Números. Revista de didáctica de las matemáticas. 80, 7-24.

Blanton, M. \& Kaput, J. (2005). Characterizing a classroom practice that promotes Algebraic reasoning. Journal for Research in Mathematics Education. 36 (5), 412-446. DOI: $10.2307 / 30034944$

Blanton, M. \& Kaput, J. (2011). Functional thinking as a route into algebra in the elementary grades. En: Cai y Knuth (Eds.) Early algebraization: A globaldialogue from multiple perspectives. Springer. DOI: 10.1007/978-3-642-17735-4

Davydov, V. (1995). Introduction to the grade 1 teacher manual.

Flores, R. (2005). El significado del algoritmo de la sustracción en la solución de problemas. Educación Matemática. 17 (2), 7-34.

Fuchs, L.; Fuchs, D.; Finelli, R.; Courey, S. \& Hamlett, C. (2004). Expanding schema-based transfer instruction to help third graders solve real-life mathematical problems. American Educational Research Journal. 41 (2), 419-445. DOI: 10.3102/00028312041002419

Fuchs, L.; Seethaler, P.; Powell, S.; Fuchs, D.; Hamlett, C. \& Fletcher, J. (2008). Effects of preventative tutoring on the mathematical problem solving of thirdgrade students with math and reading difficulties. Exceptional Children. 74, 155-173.
Fuchs, L., Zumeta, R., Finelli, R., Powell, S., Seethaler, P., Hamlett, C. \& Fuchs, D. (2010). The effects of schema-broadening instruccion on secon grades'word problem perfomance and their ability to represent word problems with algebraic equations: A randomized control study. The Elementary School Journal, 110 (4), 446463. DOI: $10.1086 / 651191$

INNE (2010). México en PISA 2009. México: INNE.

Instituto Nacional para la Evaluación de la Educación, (2013). El aprendizaje en sexto de primaria en México: Informe sobre los resultados del EXCALE 06 (2009) Español, matemáticas, ciencias naturales y educación cívica. México: INNE.

Kaput, J. (2008). What is algebra? What is algebraic reasoning? En: Kaput, Carraher y Blanton (Eds.) Algebra in the early grades.

National Council of Teachers of Mathematics (NCTM) (2000). Principles and standards for school mathematics. Recuperado de: http://www. nctm.org/uploadedFiles/Math_Standards/12752_exec_pssm.pdf

OCDE (2009). PISA 2009 Assessment framework key competencies in reading, mathematics and science. Paris: OCDE.

Schliemann, A., Carraher, D. y Brizuela, B. (2011). El carácter algebraico de la aritmética: De las ideas de los niños a las actividades en el aula. México: Paidós (Trabajo original publicado en 2007).

Secretaria de Educación Pública (2011). Programas de estudio 2011. Guía para el maestro. Educación básica primaria sexto grado. Recuperado el 18 de febrero de 2014, de http://basica.sep.gob.mx/reformaintegral/sitio/ pdf/primaria/plan/Prog6Primaria. pdf 
Secretaria de Educación Pública (2013). Evaluación Nacional de Logro Académico en Centros Escolares. Resultados históricos nacionales 2006-2013 3ro, 4to, 5to y 6to de primaria 1ro, 2do y 3ro de secundaria español, matemáticas y formación cívica y ética. Recuperado el 14 de febrero de 2014, de http://www.enlace.sep.gob.mx/content/ gr/docs/2013/historico/00_EB_2013.pdf
Tercer Estudio Regional Comparativo y Explicativo (TERCE) (2015). UNESCO. Recuperado el 25 de febrero de 2016, de http://unesdoc.unesco.org/ images/0022/002275/227501s.pdf

Vygotsky, L. (2009). Pensamiento y lenguaje. México: Ediciones Quinto sol.

\section{Apéndice 1}

Tipos de problemas algebraicos

utilizados en la investigación, retomados de literatura del área

\section{- Pensamiento relacional}

Bárbara y Juana tuvieron su fiesta de cumpleaños el mismo día. Bárbara recibió 7 regalos de sus amigos, y Juana también recibió 7 regalos de sus amigos. Cuando cada fiesta había terminado, las niñas pasaron un tiempo con sus respectivas familias y recibieron aún más regalos. Bárbara recibió 6 regalos más de su familia y Juana recibió 3 regalos más de su familia. Al final del día, ¿piensas que Juana recibió la misma cantidad de regalos que Bárbara o piensas que una recibió más regalos que la otra?

\section{- Problema de relaciones algebraicas con incógnitas}

Daniel y Kevin el día de hoy jugaron a las canicas. Al final del juego, David tenía un montón de canicas verdes y Kevin también tenía un montón de canicas verdes. Los dos montones tenían el mismo número de canicas. Daniel y Kevin contaron todas las canicas y encontraron que había 10. ¿Cuántas canicas había en cada montón?

\section{- Problema algebraico con incógnitas y variables}

Rocío y Estephani han salido a juntar manzanas para cocinar unos pasteles. Rocío juntó 7 manzanas rojas y algunas manzanas verdes. Estephani juntó 2 manzanas rojas, el mismo número de manzanas verdes que Rocío y algunas manzanas amarillas. Al final, Rocío tenía el mismo número de manzanas que Estephani. ¿Cuántas manzanas amarillas juntó Estephani? 


\section{Anexo 1}

Ejemplo de problemas aritméticos de Total de elaboración propia

\section{- Con la incógnita en el Total}

En la granja de Jacinto hay 23 puercos gordos y 39 vacas lecheras. ¿Cuántos animales hay en su granja?

\section{- Con la incógnita en la parte uno}

El profesor de arte tenía 42 piezas de papel para construir figuras. Algunos de los pedazos de papel eran azules, y 20 pedazos de papel eran verdes. ¿Cuántas piezas de papel azul tiene?

\section{- Con la incógnita en la parte dos}

Camila y Emilio tienen 28 flores entre los dos. Si Camila tiene 13, ¿cuántas flores tiene Emilio?

\section{- Con tres sumandos}

El día de muertos le dieron a Ana 27 paletas, algunos totis y 34 chocolates. $\mathrm{Al}$ llegar a su casa, Ana contó 65 dulces. ¿Cuántos totis le regalaron a Ana? 